\title{
Coherence-Multiplexed Optical RF Feeder Networks
}

\author{
Arjan Meijerink, Member, IEEE, Robert O. Taniman, Student Member, IEEE, and \\ Wim van Etten, Senior Member, IEEE
}

\begin{abstract}
An optical RF feeding system for wireless access is proposed, in which the radio access points are distinguished by means of coherence multiplexing (CM). CM is a rather unknown and potentially inexpensive optical code division multiple access technique, which is particularly suitable for relatively short-range applications with moderate transmission bandwidth requirements. Subcarrier multiplexing (SCM) can possibly be used on top of CM, either as single-channel or multichannel SCM. The performances of the resulting distribution networks are analyzed, incorporating the effect of chromatic dispersion, optical beat noise, shot noise, thermal noise, and-in the case of multichannel SCM-intermodulation distortion. The results of the analyses are illustrated by using numerical examples, based on the IEEE 802.11b standard for wireless LAN.
\end{abstract}

Index Terms-Coherence multiplexing (CM), optical code division multiple access, optical communication, radio-over-fiber (RoF) techniques, RF photonics.

\section{INTRODUCTION}

$\mathbf{R}$ ECENT interest in wireless access systems has given impetus to the development of low-cost RF feeder technologies. As an example, consider the (simplified) indoor wireless access system in Fig. 1. The high transmission frequencies in such systems (several gigahertz up to several tens of gigahertz) limit the transmission range of the radio access points (RAPs) and mobile terminals (MTs) to relatively small cells. Therefore, many RAPs are required in order to provide coverage to the MTs so that they can connect to the central node $(\mathrm{CN})$ from anywhere in the envisioned service area. Moreover, deployment density might need to be further increased in case a large number of MTs are to be supported in relatively small parts of the service area. A second issue is that modern wireless transmission requires some very complicated functions such as coders, modulators, and frequency upconverters (mixers). For presently used standards such as IEEE 802.11b [1] and 802.11g [2], the industry has managed to develop very affordable hardware in which these functions are electronically integrated. However, for future schemes (with data rates in excess of $100 \mathrm{Mb} / \mathrm{s}$ and

Manuscript received February 15, 2007; revised May 31, 2007. This work was part of the Coherence Multiplexing project supported by Philips Research, Eindhoven, The Netherlands.

A. Meijerink and W. van Etten are with the Telecommunication Engineering Group, Faculty of Electrical Engineering, Mathematics, and Computer Science, University of Twente, 7500 AE Enschede, The Netherlands (e-mail: a.meijerink@ieee.org; etten@ieee.org).

R. O. Taniman is with the Chair for Design and Analysis of Communication Systems, Faculty of Electrical Engineering, Mathematics, and Computer Science, University of Twente, 7500 AE Enschede, The Netherlands (e-mail: r.o.taniman@utwente.nl).

Digital Object Identifier 10.1109/JLT.2007.907746

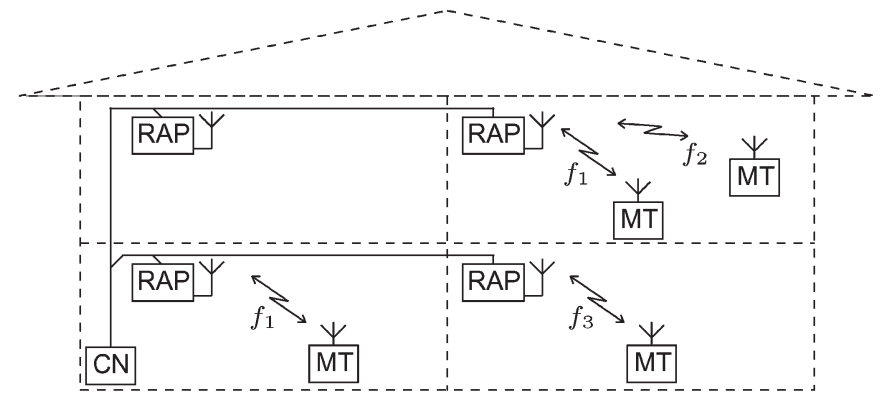

Fig. 1. Example of a simple indoor wireless access system $(\mathrm{CN}=$ central node, $\mathrm{RAP}=$ radio access point, and $\mathrm{MT}=$ mobile terminal).

radio frequencies in the $60-\mathrm{GHz}$ range), such hardware is likely to become much more expensive. Hence, installing it in the RAPs might make a wireless access system very expensive, especially when a lot of RAPs are required in order to provide coverage over a large area (for example, a large office building or an airport).

A less expensive alternative could be to concentrate these functionalities in the $\mathrm{CN}$ and, hence, convert the signals into air wave format prior to distributing them through the access network so that the $\mathrm{CN}$, in fact, acts as a remote base station. Since copper cables do not provide enough bandwidth to distribute such signals over reasonable distances, optical fiber should be used as a transmission medium. In that case, the RAPs only need to contain an opto-electronic converter and a power amplifier. This concept is widely known as radioover-fiber (RoF) transmission [3]. Besides the reduced RAP complexity, RoF shares common advantages with other optical fiber transmission techniques, such as reliability, transparency, low attenuation and dispersion, and low electromagnetic interference. Depending on the wireless transmission standard that is used and the required number of RAPs, RoF could hence result in a less expensive, more reliable, and more flexible access system with a larger network span than in the case where all the RAPs are full-featured base stations, which are connected by copper transmission media.

When part of the RF feeding network consists of a common transmission fiber, optical multiplexing is required in order to enable the $\mathrm{CN}$ to distribute signals to several RAPs at the same time, hence simultaneously serving multiple MTs. Moreover, multiplexing provides flexibility to the network, especially with respect to bandwidth assignment and network extension. Costs form an important design criterion for such an optically multiplexed distribution network.

In this paper, an optical RF feeder technology is proposed, based on an optical code division multiplexing technique, 


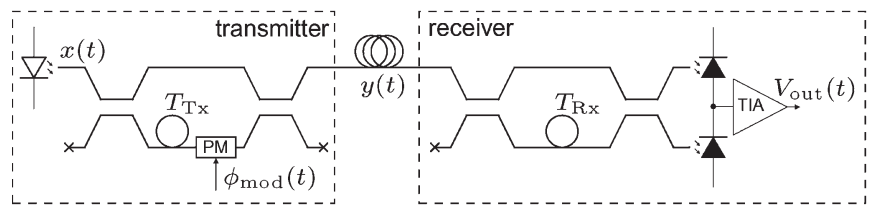

Fig. 2. Simple CM system with one transmitter and one (balanced) receiver $(\mathrm{PM}=$ phase modulator and TIA $=$ transimpedance amplifier $)$.

which is known as coherence multiplexing (CM) [4]-[18]. Because of its potentially simple implementation, CM is an interesting candidate to distinguish between the RAPs. Moreover, subcarrier multiplexing (SCM) could be used on top of CM [11], [12], [15], [18] (for example, to support multiple MTs through the same RAP or support multicarrier modulation formats).

This paper is organized as follows. In the next section, channel generation and multiplexing and demultiplexing principles of CM will be briefly summarized. In Section III, an optical RF distribution network based on CM will be introduced, and Section IV will describe how single-channel and multichannel SCM can be used on top of CM in such a system. The systems in both sections will be analyzed with respect to chromatic dispersion, noise, and intermodulation distortion (IMD), and the results of these analyses will be illustrated by using numerical examples, based on the IEEE $802.11 \mathrm{~b}$ wireless LAN standard [1]. This paper ends with the conclusion in Section V.

\section{CM PRINCIPLES}

$\mathrm{CM}$ is a rather unknown optical code division multiple access technique, although it has existed for almost 30 years [4]-[18]. It is based on a channel generation technique called coherence modulation, which was introduced by Delisle and Cielo [4]. It is shown in Fig. 2, which shows one coherence modulator (transmitter) and demodulator (receiver). The transmitter consists of a broadband optical source and a Mach-Zehnder interferometer (MZI) with path delay difference (PDD) $T_{\mathrm{Tx}}$, which is chosen to be much larger than the coherence time of the source signal. Hence, there is no coherent beating (interference) so that the modulating signal $\phi_{\text {mod }}(t)$ does not result in an observable intensity modulation in the output signal $y(t)$ of the transmitter. The modulating signal can be made observable at the receiver by means of interference, using an MZI with a PDD $T_{\mathrm{Rx}}$ that is equal to $T_{\mathrm{Tx}}$. On the other hand, $\phi_{\text {mod }}(t)$ will not be observable when the difference between $T_{\mathrm{Rx}}$ and $T_{\mathrm{Tx}}$ is much larger than the coherence time of the source signal, because there is no interference in that case. Hence, the relation between the PDDs in the transmitter $\left(T_{\mathrm{Tx}}\right)$ and receiver $\left(T_{\mathrm{Rx}}\right)$ determines whether a coherence-modulated channel is demodulated or not. Although a balanced receiver is considered in Fig. 2, the desired channel can also be demodulated by means of a single photodiode at one MZI output, which is, in fact, what Delisle and Cielo did. However, a balanced receiver does not require dc cancellation and can be shown to have a superior noise performance [13]. Moreover, balanced detection enables the phase modulator in the transmitter's MZI to be replaced by

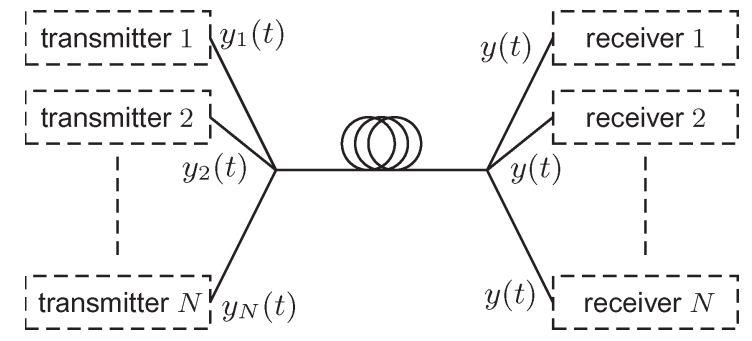

Fig. 3. Coherence-multiplexed optical network with $N$ transmitters and $N$ receivers in a parallel array configuration.

an intensity modulator in front of the MZI, but that will not be further considered here.

Cielo and Delisle [5] extended their work by multiplexing several coherence-modulated channels, using cascaded coherence modulators with a common light source. Several alternative multiplexing topologies using common sources were proposed by Brooks et al. [6] and analyzed by Wentworth [9], within the framework of optical interferometric sensors. Later on Goedgebuer and Hamel introduced the parallel array, in which each coherence modulator has its own light source [8], [10], [13], [14]. In this paper, we will only consider the parallel array, as it provides most flexibility with respect to transmitter location [17], [18]. A parallel array topology with $N$ transmitters and $N$ receivers is shown in Fig. 3. Each transmitter $i$ launches one coherence-modulated optical signal $y_{i}(t)$ into the common transmission fiber. Each receiver receives a similar composite signal $y(t)$ and only demodulates the data signal coming from the coherence modulator that has the same PDD value. Channel crosstalk is hence prevented if the PDDs of the MZIs in the transmitters have mutual differences that are much larger than the coherence time of the optical signals. CM has the following main advantages with respect to other multiplexing techniques.

1) Relatively simple optical sources such as light-emitting diodes (LEDs) or superluminescent LEDs can be used as broadband optical sources, and channel generation and selection can be performed by simple MZIs, which can be integrated as, for example, planar optical waveguide circuits.

2) The channel selection concept is relatively robust in the sense that temperature fluctuations will not result in crosstalk from undesired channels.

3) The transmission concept is transparent, and the channels can independently be operated so that $\mathrm{CM}$ can be used for both digital and analog transmissions, possibly simultaneously, without mutual synchronization between the transmitters.

However, CM also has the following particular disadvantages.

1) The optical beating of mutually incoherent signals in the receiver results in optical beat interference noise (or beat noise). Its power increases with an increasing number of active channels.

2) The large bandwidth of the optical source signal makes the modulated transmitted optical signal vulnerable to chromatic dispersion in the transmission fiber.

3) The transmitted optical power is shared by all receivers. 
These disadvantages fundamentally limit the number of CM channels that can be multiplexed, the data signal bandwidths that can be supported, and the transmission distances that can be spanned. Therefore, CM seems to be particularly suitable for low-cost relatively short-range applications with moderate transmission bandwidth requirements, such as subscriber networks, local area networks, and interconnects.

Another issue in CM systems is that the phases and polarization states of the interfering signals have to be matched in order to maximize the amplitude of the output signal of the receiver. Polarization will not be an issue in the case where integrated MZIs are used for modulation and demodulation, but there might still be a phase offset due to small differences between $T_{\mathrm{Tx}}$ and $T_{\mathrm{Rx}}$ (for example, due to fabrication inaccuracies, temperature drift, or aging). Several solutions are known in curing this problem (for example, phase synchronization techniques using a feedback loop, or phase diversity [17], [18]).

\section{Coherence-Multiplexed Optical RF FEEDER NETWORK}

\section{A. System Description and Assumptions}

This paper deals with CM systems in which the modulating signals are RF signals. First, consider the simple case where each CM channel conveys only one RF signal. These channels are multiplexed in a CM system with a parallel array topology, consisting of $N$ transmitters and $N$ balanced receivers (see Figs. 2 and 3). Each transmitter $i$ consists of an MZI with PDD $T_{\mathrm{Tx}, i}$ and modulating signal

$$
\phi_{\bmod , i}(t)=\beta_{i}(t) \sin \left(2 \pi f_{i} t+\psi_{i}(t)\right)
$$

where $\beta_{i}(t), f_{i}$, and $\psi_{i}(t)$ are the amplitude, carrier frequency, and phase of the modulating RF signal in transmitter $i$, respectively. The behavior of $\beta_{i}(t)$ and $\psi_{i}(t)$ depends on the $\mathrm{RF}$ signal format, which is, for example, prescribed by a wireless transmission standard. The MZI is illuminated by a broadband optical source with average optical power $P_{\text {in }}$, center frequency $f_{\mathrm{c}}$, and coherence time $\tau_{\mathrm{c}}$. Each receiver $r$ is assumed to be matched to the corresponding transmitter $r$ on a coherence time scale (so $\left|T_{\mathrm{Rx}, r}-T_{\mathrm{Tx}, r}\right| \ll \tau_{\mathrm{c}} \forall r$ and $\mid T_{\mathrm{Rx}, r}-$ $T_{\mathrm{Tx}, i} \mid \gg \tau_{\mathrm{c}}$ for $r \neq i$ ). All directional couplers are assumed to be perfectly balanced, and the photodiodes in the balanced receivers are assumed to linearly operate and to have identical responsivities $R_{\mathrm{pd}}$. Transimpedance amplifiers (TIAs) have a transimpedance $Z_{\text {TIA }}$ and are followed by a bandpass filter with an impulse response $h_{\mathrm{BP}, r}(t)$. The excess losses in the transmitter and receiver (due to device and fiber-chip coupling losses) are denoted by $L_{\mathrm{Tx}}$ and $L_{\mathrm{Rx}}$, respectively, and $L_{\mathrm{nw}}$ denotes the optical losses in the network (due to propagation losses and coupling and splitting losses). Chromatic dispersion in the transmission fiber is initially ignored; we will consider this in Section III-D.

\section{B. Receiver Output Signal Analysis}

With the assumptions in the previous section, it can be proven that the expected value of the output signal of the bandpass filter in receiver $r$ is given by

$$
\begin{aligned}
& E\left[V_{\mathrm{BP}, r}(t)\right] \approx \frac{Z_{\mathrm{TIA}} R_{\mathrm{pd}} P_{\mathrm{in}}}{4 L_{\mathrm{Tx}} L_{\mathrm{Rx}} L_{\mathrm{nw}}} \\
& \cdot \int h_{\mathrm{BP}, r}(t-\rho) \cdot \cos \left(\Delta \phi_{r}+\phi_{\bmod , r}(\rho)\right) d \rho
\end{aligned}
$$

where $\Delta \phi_{r} \triangleq 2 \pi f_{\mathrm{c}}\left(T_{\mathrm{Rx}, r}-T_{\mathrm{Tx}, r}\right)$ is the phase offset between the interfering optical signals due to small mismatches between the PDDs in the transmitter and receiver $r$. By factorizing the cosine in (2), by substituting (1), and by expanding the resulting nested sines and cosines into a Fourier series, we can write

$$
\begin{gathered}
\mathrm{E}\left[V_{\mathrm{BP}, r}(t)\right]=\frac{Z_{\mathrm{TIA}} R_{\mathrm{pd}} P_{\mathrm{in}}}{4 L_{\mathrm{Tx}} L_{\mathrm{Rx}} L_{\mathrm{nw}}} \int h_{\mathrm{BP}, r}(t-\rho) \\
\cdot\left\{\operatorname { c o s } ( \Delta \phi _ { r } ) \left[J_{0}\left(\beta_{r}(\rho)\right)+2 \sum_{\substack{n=2 \\
n \text { even }}}^{\infty} J_{n}\left(\beta_{r}(\rho)\right)\right.\right. \\
\left.\cdot \cos \left(2 \pi n f_{r} \rho+n \psi_{r}(\rho)\right)\right] \\
-2 \sin \left(\Delta \phi_{r}\right) \sum_{\substack{n=1 \\
n \text { odd }}}^{\infty} J_{n}\left(\beta_{r}(\rho)\right) \\
\left.\cdot \sin \left(2 \pi n f_{r} \rho+n \psi_{r}(\rho)\right)\right\} d \rho
\end{gathered}
$$

where the Fourier coefficients $J_{n}(\cdot)$ are Bessel functions of the first kind and order $n$

$$
J_{n}(x) \triangleq \frac{1}{\pi} \int_{0}^{\pi} \cos (x \sin \theta-n \theta) d \theta .
$$

Apparently, harmonic distortion occurs due to the intrinsic nonlinearity of the demodulation principle. As a result, the output signal contains a baseband term and an infinite sum of RF terms with carrier frequencies that are integer multiples of $f_{r}$. The desired part is the RF term with carrier frequency $f_{r}$. Hence, the bandpass filter should be dimensioned such that this term is passed, and the other terms are suppressed. The amplitude of the resulting signal can be maximized by setting $\Delta \phi_{r}=-(1 / 2) \pi$ (we will consider this in Section III-C), resulting in

$$
\begin{aligned}
E\left[V_{\mathrm{BP}, r}(t)\right]=\frac{Z_{\mathrm{TIA}} R_{\mathrm{pd}} P_{\mathrm{in}}}{2 L_{\mathrm{Tx}} L_{\mathrm{Rx}} L_{\mathrm{nw}}}\left|H_{\mathrm{BP}, r}\left(f_{r}\right)\right| \\
\cdot J_{1}\left(\beta_{r}(t)\right) \sin \left(2 \pi f_{r} t+\psi_{r}(t)\right)
\end{aligned}
$$

where $H_{\mathrm{BP}, r}(f)$ is the transfer function of the bandpass filter. An irrelevant phase shift due to the filter has been omitted.

$J_{1}(\cdot)$ is a nonlinear function. Hence, when the RF feeder system is used in an application where the amplitude $\beta_{r}(t)$ of the RF signal is modulated by an analog signal (for example, in analog TV distribution) or by means of a multilevel digital modulation technique (such as $M$-ary pulse-amplitude or quadraticamplitude modulation), then a predistortion circuit should be 
used, or $\beta_{r}(t)$ should be kept small such that $J_{1}\left(\beta_{r}(t)\right) \approx$ $\beta_{r}(t) / 2$. However, when $\beta_{r}(t)$ is a constant $\beta$ (or, possibly, when $\beta_{r}(t)$ only takes the values 0 and $\beta$ ), then the amplitude of the output signal can be maximized by choosing $\beta$ such that $J_{1}(\beta)$ is maximized. By numerically solving for the zero of the derivative $J_{1}^{\prime}(\beta)=J_{0}(\beta)-J_{1}(\beta) / \beta$, it can be found that this is the case when $\beta \approx 1.84$, which gives $J_{1}(\beta) \approx 0.58$. The output signal with maximum amplitude is, hence, given by

$$
\begin{aligned}
E\left[V_{\mathrm{BP}, r}(t)\right] \approx 0.29 & \frac{Z_{\mathrm{TIA}} R_{\mathrm{pd}} P_{\mathrm{in}}}{L_{\mathrm{Tx}} L_{\mathrm{Rx}} L_{\mathrm{nw}}} \\
& \cdot\left|H_{\mathrm{BP}, r}\left(f_{r}\right)\right| \sin \left(2 \pi f_{r} t+\psi_{r}(t)\right) .
\end{aligned}
$$

\section{Output Signal Stabilization}

It follows from (3) that the amplitude of the output signal is proportional to $-\sin \left(\Delta \phi_{r}\right)$. Hence, the value of $\Delta \phi_{r}$ should be locked to $-(1 / 2) \pi$ in order to obtain a stable maximum output signal amplitude. A suitable control signal for doing this can be extracted from the baseband term in (3). This term can be obtained by applying the input signal of the bandpass filter to a low-pass filter, which has an impulse response $h_{\mathrm{LP}}(t)$ such that all the RF terms are suppressed. The output signal of this filter then follows from (3) as

$$
\begin{aligned}
E\left[V_{\mathrm{LP}, r}(t)\right]= & \frac{Z_{\mathrm{TIA}} R_{\mathrm{pd}} P_{\mathrm{in}}}{4 L_{\mathrm{Tx}} L_{\mathrm{Rx}} L_{\mathrm{nw}}} \\
& \cdot \cos \left(\Delta \phi_{r}\right) \int h_{\mathrm{LP}}(t-\rho) J_{0}\left(\beta_{r}(\rho)\right) d \rho .
\end{aligned}
$$

If the low-pass filter also sufficiently suppresses temporal variations in $\beta_{r}(t)$ (or if $\beta_{r}(t)$ is simply a constant $\beta$ ), the output signal becomes proportional to $\cos \left(\Delta \phi_{r}\right)$. Since $\Delta \phi_{r}=$ $2 \pi f_{\mathrm{c}}\left(T_{\mathrm{Rx}, r}-T_{\mathrm{Tx}, r}\right)$, it follows that proper phase synchronization can be achieved by means of a feedback loop, which slightly increases the value of $T_{\mathrm{Rx}, r}$ when $V_{\mathrm{LP}, r}(t)$ is positive and decreases $T_{\mathrm{Rx}, r}$ when $V_{\mathrm{LP}, r}(t)$ is negative. Note that this does not require any form of frequency dithering.

An alternative is to use phase diversity techniques: Some schemes for digital CM receivers were proposed in [17] and extended to RF receivers in [18], but these will not be considered further here.

\section{Effect of Chromatic Dispersion}

Due to the relatively large bandwidth of the optical signal, the output signal of the receiver will be distorted by the chromatic dispersion in the transmission fiber. This effect was studied for digital transmission over CM by Pendock and Sampson [13]. Their analysis is based on the paper by Gimlett and Cheung [19], in which dispersion penalties are calculated for optical transmission systems using LEDs, single-mode fiber (SMF), intensity modulation, and direct detection. They derive that the impact of the chromatic dispersion on the receiver output signal can be modeled as the insertion of an electrical linear timeinvariant low-pass filter with transfer function $H_{\mathrm{CD}}(f)$. In our case, where the output signal is an RF bandpass signal, this will result in carrier amplitude fading. The RF power reduction factor can be found by calculating the squared magnitude of $H_{\mathrm{CD}}(f)$ at the RF carrier frequency $f_{r}$, resulting in

$$
\left|H_{\mathrm{CD}}\left(f_{r}\right)\right|^{2}=\frac{1}{\sqrt{1+f_{r}^{2} T_{2}^{2}}} \exp \left(-\frac{f_{r}^{2} T_{1}^{2}}{1+f_{r}^{2} T_{2}^{2}}\right)
$$

where

$$
\begin{aligned}
& T_{1}=2 \pi l_{\mathrm{f}} \sigma_{\lambda} D \\
& T_{2}=2 \pi l_{\mathrm{f}} \sigma_{\lambda}^{2} D
\end{aligned}
$$

where $D_{1}$ and $D_{2}$ are the first- and second-order dispersion coefficients, respectively, $l_{\mathrm{f}}$ is the length of the fiber, and $\sigma_{\lambda}$ is the rms width of the (Gaussian) power spectral density function of the optical field.

In practice, either first-order dispersion $\left(T_{1}\right)$ or second-order dispersion $\left(T_{2}\right)$ will be dominant, depending on the center wavelength $\lambda_{\mathrm{c}}$ and the fiber type.

\section{E. Noise Analysis}

Apart from chromatic dispersion, the output signal of the receiver is also corrupted by noise. Three types of noise will be considered here.

1) source-induced noise, which is caused by the random behavior of the optical source signal, resulting in random fluctuations in the power of the detected signal. Source intensity noise results in random fluctuations of the desired interference term, and source phase noise results into beat noise, as mentioned in Section II;

2) shot noise, which is due to the random arrival times of photons;

3) thermal receiver noise, which is caused by the random motion of electrons and holes in the electronic components.

The noise performance of CM systems has actually been extensively studied over the years, with most publications relying on Wentworth's model [9]. His performance analysis on various multiplexing topologies was extended to the parallel array by Chu and Dickey [10]. However, their results were limited to single-ended receivers. In [13], a parallel array with balanced receivers is studied, but shot and thermal noises are ignored so that the results only apply for sufficiently high received powers. By combining the various existing approaches, the noise variance at the output of the receiver's bandpass filter is given as [17], [18]

$$
\begin{aligned}
\sigma_{V_{\mathrm{BP}, r}(t)}^{2} \approx & \frac{Z_{\mathrm{TIA}}^{2} R_{\mathrm{pd}}^{2} P_{\mathrm{in}}^{2} \tau_{\mathrm{c}}}{32 L_{\mathrm{Tx}}^{2} L_{\mathrm{Rx}}^{2} L_{\mathrm{nw}}^{2}} \\
& \cdot \int h_{\mathrm{BP}, r}^{2}(\rho)\left[4 N^{2}+2 N-1\right. \\
& \left.\quad+2 \cos ^{2}\left(\Delta \phi_{r}+\phi_{\mathrm{mod}, r}(t-\rho)\right)\right] d \rho \\
& +\frac{Z_{\mathrm{TIA}}^{2} R_{\mathrm{pd}} P_{\mathrm{in}} e N}{2 L_{\mathrm{Tx}} L_{\mathrm{Rx}} L_{\mathrm{nw}}} \int h_{\mathrm{BP}, r}^{2}(\rho) d \rho \\
& +Z_{\mathrm{TIA}}^{2} S_{\mathrm{th}} \int h_{\mathrm{BP}, r}^{2}(\rho) d \rho
\end{aligned}
$$


where $e$ is the charge of an electron $\left(1.60 \cdot 10^{-19} \mathrm{C}\right)$, and $S_{\mathrm{th}}$ is the power spectral density of the equivalent input noise current to the TIA. With the definition that Wentworth [9] used, the coherence time $\tau_{\mathrm{c}}$ is related to the rms width $\sigma_{\lambda}$ by

$$
\tau_{\mathrm{c}}=\frac{1}{2 \sqrt{\pi}} \frac{\lambda_{\mathrm{c}}^{2}}{c_{0} \sigma_{\lambda}}
$$

where $\lambda_{\mathrm{c}}$ is the optical center wavelength, and $c_{0}$ is the speed of light in a vacuum.

The three terms in (11) correspond to source-induced, shot, and thermal noises. By substituting $\Delta \phi_{r}=-(1 / 2) \pi$ and (1) and by assuming that the amplitude $\beta_{r}(t)$ of the modulating signal is small, the $\cos ^{2}(\cdot)$ term in (11) (which corresponds to the source intensity-induced noise) can be neglected. The integral can be calculated by writing the impulse response $h_{\mathrm{BP}, r}(t)$ as the inverse Fourier transform of the transfer function $H_{\mathrm{BP}, r}(f)$ of the bandpass filter. We then find that (11) can be written as

$$
\begin{aligned}
\sigma_{V_{\mathrm{BP}, r}(t)}^{2} \approx & 2 Z_{\mathrm{TIA}}^{2}\left[\frac{R_{\mathrm{pd}}^{2} P_{\mathrm{in}}^{2}\left(4 N^{2}+2 N-1\right) \tau_{\mathrm{c}}}{32 L_{\mathrm{Tx}}^{2} L_{\mathrm{Rx}}^{2} L_{\mathrm{nw}}^{2}}\right. \\
& \left.+\frac{R_{\mathrm{pd}} P_{\mathrm{in}} e N}{2 L_{\mathrm{Tx}} L_{\mathrm{Rx}} L_{\mathrm{nw}}}+S_{\mathrm{th}}\right]\left|H_{\mathrm{BP}, r}\left(f_{r}\right)\right|^{2} W_{\mathrm{BP}}
\end{aligned}
$$

where $W_{\mathrm{BP}}$ is the equivalent noise bandwidth of the bandpass filter

$$
W_{\mathrm{BP}} \triangleq \frac{1}{\left|H_{\mathrm{BP}, r}\left(f_{r}\right)\right|^{2}} \int_{0}^{\infty}\left|H_{\mathrm{BP}, r}(f)\right|^{2} d f .
$$

The average carrier-to-noise ratio at the output of the bandpass filter now follows from (5), (8), and (13) as

$$
\begin{aligned}
\mathrm{CNR} & =\frac{\left\langle E^{2}\left[V_{\mathrm{BP}, r}(t)\right]\right\rangle\left|H_{\mathrm{CD}}\left(f_{r}\right)\right|^{2}}{\sigma_{V_{\mathrm{BP}, r}(t)}^{2}} \\
& =\left(\mathrm{CNR}_{\mathrm{bn}}^{-1}+\mathrm{CNR}_{\mathrm{sn}}^{-1}+\mathrm{CNR}_{\mathrm{tn}}^{-1}\right)^{-1}
\end{aligned}
$$

where $\langle\cdot\rangle$ denotes time-averaging, and

$$
\begin{aligned}
\mathrm{CNR}_{\mathrm{bn}} & =\frac{2\left\langle J_{1}^{2}\left(\beta_{r}(t)\right)\right\rangle\left|H_{\mathrm{CD}}\left(f_{r}\right)\right|^{2}}{\left(4 N^{2}+2 N-1\right) \tau_{\mathrm{c}} W_{\mathrm{BP}}} \\
\mathrm{CNR}_{\mathrm{sn}} & =\frac{R_{\mathrm{pd}} P_{\mathrm{in}}\left\langle J_{1}^{2}\left(\beta_{r}(t)\right)\right\rangle\left|H_{\mathrm{CD}}\left(f_{r}\right)\right|^{2}}{8 N L_{\mathrm{Tx}} L_{\mathrm{Rx}} L_{\mathrm{nw}} e W_{\mathrm{BP}}} \\
\mathrm{CNR}_{\mathrm{tn}} & =\frac{R_{\mathrm{pd}}^{2} P_{\mathrm{in}}^{2}\left\langle J_{1}^{2}\left(\beta_{r}(t)\right)\right\rangle\left|H_{\mathrm{CD}}\left(f_{r}\right)\right|^{2}}{16 L_{\mathrm{Tx}}^{2} L_{\mathrm{Rx}}^{2} L_{\mathrm{nw}}^{2} S_{\mathrm{th}} W_{\mathrm{BP}}}
\end{aligned}
$$

are the carrier-to-beat, carrier-to-shot, and carrier-to-thermal noise ratios, respectively.

\section{F. Numerical Example: IEEE 802.11b}

As a numerical example, suppose that the proposed concept is applied to the distribution of $11-\mathrm{Mb} / \mathrm{s}$ wireless LAN signals in an indoor wireless access network, according to the IEEE 802.11b standard [1]. Considering the prices of currently sold RAPs - in which the 802.11 b functionality is fully integrated in the RAP - this might not be the most obvious application from an economical point of view. However, we chose to use $802.11 \mathrm{~b}$ as an example as it provides a simple way to illustrate the developed theory. Standards using orthogonal frequency division multiplexing (OFDM) would require more complicated analysis of the IMD between the different carriers. We will get back to this in the conclusion of this paper.

Only the downlink path (from the CN to the RAPs) is considered. Each RAP is assigned one CM channel so that there are $N \mathrm{CM}$ transmitters (in the $\mathrm{CN}$ ), an $N \times 1$ optical combiner, a $1 \times N$ optical splitter, and $N$ RAPs, where each RAP contains one $\mathrm{CM}$ receiver.

IEEE 802.11 b operates in the $2.4-\mathrm{GHz}$ industrial, scientific, and medical band, and in the $11-\mathrm{Mb} / \mathrm{s}$ mode, it uses complementary code keying modulation as a direct-sequence spreadspectrum technique, with a chip rate of $11 \mathrm{MHz}$. The coded chips are transmitted using QPSK modulation. The resulting effective channel bandwidth after spectral shaping is approximately $17 \mathrm{MHz}$.

The optical sources are assumed to couple $P_{\text {in }}=10 \mathrm{~mW}$ of optical power into each MZI, and the center wavelength $\lambda_{\mathrm{c}}$ is initially assumed to be $1550 \mathrm{~nm}$. This is a common wavelength in fiber optics as this gives the lowest attenuation in standard SMF [20]. The coherence time $\tau_{\mathrm{c}}$ is assumed to be $0.1 \mathrm{ps}$. By using (12), it can be verified that this corresponds to an rms linewidth of $23 \mathrm{~nm}$ when $\lambda_{\mathrm{c}}=1550 \mathrm{~nm}$.

Since QPSK modulation is used in IEEE 802.11b, the modulating signals $\phi_{\bmod , i}(t)$ have a constant amplitude $\beta_{i}(t)=\beta$. In order to maximize the receiver's output signal amplitude, the optimal value $\beta=1.84$ is used, as discussed previously.

For the transmission fiber, consider a standard SMF with a typical indoor length $l_{\mathrm{f}}=500 \mathrm{~m}$. The effect of chromatic dispersion then depends on the optical center wavelength $\lambda_{c}$. When $\lambda_{\mathrm{c}}=1550 \mathrm{~nm}$, second-order dispersion can be neglected $\left(T_{2} \ll T_{1}\right)$, and the first-order dispersion coefficient $D_{1}$ is prescribed to have a maximum value of $20 \mathrm{ps} /(\mathrm{nm} \cdot \mathrm{km})$ [20]. By using (9), one can find that $T_{1} \approx 1.4 \mathrm{~ns}$ so that the RF power reduction factor follows from $(8)$ as $\left|H_{\mathrm{CD}}\left(f_{r}\right)\right|^{2} \approx 9.1 \cdot 10^{-6}$. Hence, chromatic dispersion degrades the CNR by roughly $50 \mathrm{~dB}$ when $\lambda_{\mathrm{c}}=1550 \mathrm{~nm}$, so this is not a suitable center wavelength when no dispersion compensation is used.

Alternatively, consider the so-called zero-dispersion wavelength (approximately $1310 \mathrm{~nm}$ for a standard SMF). By definition, there is no first-order dispersion at this wavelength (theoretically, $D_{1}=0$ ), and the second-order dispersion coefficient $D_{2}$ is prescribed to have a maximum value of $0.093 \mathrm{ps} /\left(\mathrm{nm}^{2} \cdot \mathrm{km}\right)$ [20]. By using (10) and (12), one can find that $\sigma_{\lambda} \approx 16 \mathrm{~nm}$ and $T_{2} \approx 76 \mathrm{ps}$. In practice, however, the exact value of $\lambda_{\mathrm{c}}$ in which $D_{1}=0$ might slightly vary from fiber to fiber. The standard prescribes it to be between 1300 and $1324 \mathrm{~nm}$, so by assuming that 1310-nm sources are used, one can assume that $D_{1} \approx 0.093 \cdot 14 \approx 1.3 \mathrm{ps} /(\mathrm{nm} \cdot \mathrm{km})$ or $T_{1} \approx 66 \mathrm{ps}$ as a rough upper bound. The RF power reduction factor now follows from $(8)$ as $\left|H_{\mathrm{CD}}\left(f_{r}\right)\right|^{2} \approx 0.96$. Hence, chromatic dispersion degrades the CNR by, at most, $0.18 \mathrm{~dB}$ when $\lambda_{\mathrm{c}}=1310 \mathrm{~nm}$ is used. 


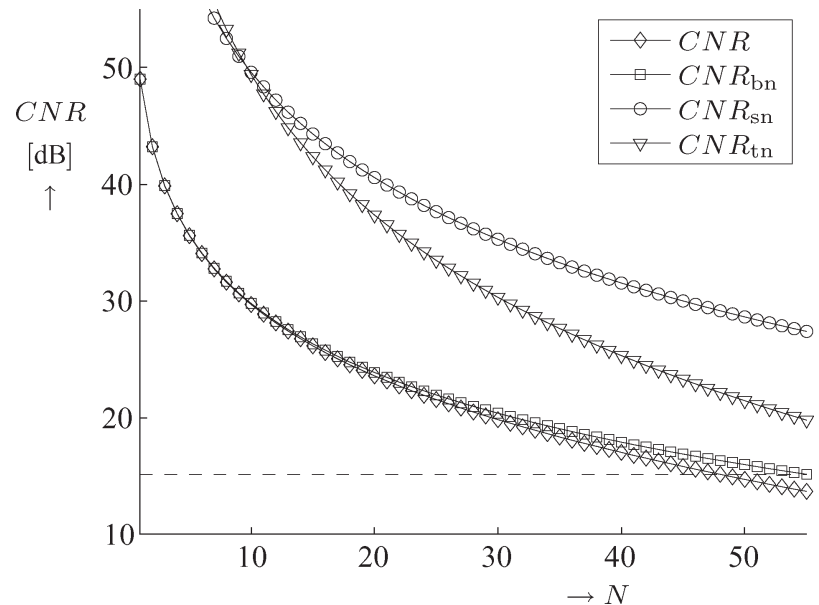

Fig. 4. Carrier-to-noise ratios at the output of the bandpass filter as a function of the number of RAPs $N$ in a wireless LAN IEEE 802.11b distribution network based on CM.

The propagation loss at $1310 \mathrm{~nm}$ will be slightly higher than at $1550 \mathrm{~nm}$ : roughly $0.35 \mathrm{~dB} / \mathrm{km}$. The splitters and combiners have an intrinsic splitting loss $N$ and are assumed to have an excess loss of $0.1 \mathrm{~dB}$. Hence, the total network losses can be calculated to be $L_{\mathrm{nw}} \approx 1.09 \mathrm{~N}^{2}$. When the MZIs in transmitters and receivers are assumed to have an excess loss of $0.5 \mathrm{~dB}$, we have $L_{\mathrm{Tx}}=L_{\mathrm{Rx}} \approx 1.12$.

The photodiodes in the optical receivers are assumed to have a responsivity $R_{\mathrm{pd}} \approx 0.8 \mathrm{~A} / \mathrm{W}$, and the TIAs are assumed to have an rms equivalent input current in the order of $7 \mathrm{pA} / \sqrt{\mathrm{Hz}}$ so that we have $S_{\mathrm{th}} \approx 5 \cdot 10^{-23} \mathrm{~A}^{2} / \mathrm{Hz}$.

The CNR at the output of the bandpass filter can now be calculated as a function of the number of channels $N$. The results are shown in Fig. 4.

The IEEE $802.11 \mathrm{~b}$ standard prescribes the radiated signals of the RAPs in the $11-\mathrm{Mb} / \mathrm{s}$ mode to have a maximum error vector magnitude of $35 \%$ [1], which corresponds to a CNR of $9 \mathrm{~dB}$. Using a rough safety margin of $6 \mathrm{~dB}$ for other impairments in the system, this results in a minimum CNR of $15 \mathrm{~dB}$, which is shown by the dashed line in Fig. 4. By using this requirement, it follows that, at most, 48 RAPs can be supported using this RF distribution concept and that this number is mainly determined by the amount of beat noise that arises when $48 \mathrm{CM}$ channels are simultaneously active.

The number of RAPs can further be increased by simply increasing the number of CM transmitters and receivers and by switching OFF all optical sources in transmitters that correspond to inactive RAPs. Hence, the maximum number of MTs that can simultaneously be supported in such a system is smaller than the number of RAPs. However, increasing the number of RAPs will also increase the combining and splitting loss in the optical network so that thermal noise will become a limiting factor. This can be compensated by using CM transmitters with tunable PDDs so that each transmitter can change to which receiver it addresses. The number of transmitters can then be the same as the maximum number of active RAPs, which reduces the combining loss in the network.

Another option in increasing the number of RAPs is to use the so-called single intrinsic reference ladder system [17], [18] as CM topology since this one has a superior carrier-to-beat noise ratio compared to the parallel array. However, this can only be used in the downlink because it relies on a common transmission unit, which has to be localized to a single node.

\section{SCM OVER CM}

Multiple RF signals can be frequency-multiplexed in each CM channel by performing SCM on top of CM [11], [12], [15], [18]. There can be several reasons for doing this.

1) Since assigning individual frequencies to individual RAPs provides another way of distinguishing between RAPs, the number of CM channels can be reduced by multiplexing several RF signals per $\mathrm{CM}$ channel. This can reduce the number of required optical transmitters for the downlink and/or the number of receivers for the uplink, resulting in cost reduction and performance improvement through reduced optical splitting/combining losses and possible beat noise reduction.

2) Multiple MTs can be supported through the same RAP if they transmit at different RF carrier frequencies. CM then provides the means to distinguish between the RAPs, whereas SCM provides the means to distinguish between the MTs that communicate through the same RAP. This is also shown in Fig. 1.

3) Multicarrier modulation formats such as OFDM (among others used in IEEE 802.11g [2]) require simultaneous transmission of different RF carriers through the same CM channel.

SCM can be performed on top of CM in two ways:

1) by transmitting different subcarriers by using separate coherence modulators that have the same PDD $T_{\mathrm{Tx}}$ (single-channel SCM);

2) by first combining the subcarriers in the electrical domain and then modulating the composite signal onto an optical carrier by means of one coherence modulator (multichannel SCM).

These two alternatives will be discussed in the following two sections.

\section{A. Single-Channel SCM}

Suppose that $K \mathrm{RF}$ signals are transmitted through the same CM channel by means of a single-channel SCM. When there are $N$ CM channels, this requires $N \cdot K$ coherence modulators, where each transmits one RF signal. The coherence modulators are assumed to be configured in a parallel array topology and can be divided in $N$ groups of $K$ coherence modulators transmitting in the same CM channel. When the MZI of the coherence modulator that corresponds to the $k$ th SCM channel in the $i$ th $\mathrm{CM}$ channel has PDD $T_{\mathrm{Tx}, i, k}$, then two PDDs corresponding to the same CM channel $i$ should be equal on a coherence time scale: $\left|T_{\mathrm{Tx}, i, k}-T_{\mathrm{Tx}, i, l}\right| \ll \tau_{\mathrm{c}}$. The modulating signals in the coherence modulators can be written as

$$
\phi_{\bmod , i, k}(t)=\beta_{i, k}(t) \sin \left(2 \pi f_{i, k} t+\psi_{i, k}(t)\right)
$$


where $\beta_{i, k}(t), f_{i, k}$, and $\psi_{i, k}(t)$ are the amplitude, carrier frequency, and phase of the $k$ th SCM channel in the $i$ th CM channel, respectively. The SCM channel frequency sets are not necessarily the same for each CM channel.

Suppose that the composite optical signal $y(t)$ is received by $N$ balanced CM receivers, where each receiver $r$ has PDD $T_{\mathrm{Rx}, r} \approx T_{\mathrm{Tx}, i, k}$ and $K$ parallel bandpass filters at the output of the TIA with impulse responses $h_{\mathrm{BP}, r, k}(t)$. Then-with similar assumptions as in Section III-A-it can be shown that the expected output signal of the $j$ th bandpass filter of receiver $r$ can be written as

$$
\begin{aligned}
\mathrm{E}\left[V_{\mathrm{BP}, r, j}(t)\right]= & \frac{Z_{\mathrm{TIA}} R_{\mathrm{pd}} P_{\mathrm{in}}}{4 L_{\mathrm{Tx}} L_{\mathrm{Rx}} L_{\mathrm{nw}}} \sum_{k=1}^{K} \int h_{\mathrm{BP}, r, k}(t-\rho) \\
& \cdot\left\{\operatorname { c o s } ( \Delta \phi _ { r , k } ) \left[J_{0}\left(\beta_{r, k}(\rho)\right)+2 \sum_{\substack{n=2 \\
n \text { even }}}^{\infty} J_{n}\left(\beta_{r, k}(\rho)\right)\right.\right. \\
& \left.\cdot \cos \left(2 \pi n f_{r, k} \rho+n \psi_{r, k}(\rho)\right)\right] \\
& -2 \sin \left(\Delta \phi_{r} k\right) \cdot \sum_{\substack{n=1 \\
n \text { odd }}}^{\infty} J_{n}\left(\beta_{r, k}(\rho)\right) \\
& \left.\cdot \sin \left(2 \pi n f_{r, k} \rho+n \psi_{r, k}(\rho)\right)\right\} d \rho
\end{aligned}
$$

where $\Delta \phi_{r, k} \triangleq 2 \pi f_{\mathrm{c}}\left(T_{\mathrm{Rx}, r}-T_{\mathrm{Tx}, r, k}\right)$ is the phase offset due to small mismatches between the PDDs in receiver $r$ and the transmitter that corresponds to $\mathrm{CM}$ channel $r$ and $\mathrm{SCM}$ channel $k$.

There is still harmonic distortion in each SCM channel, but note that there is no IMD because transmission in different optical carriers prevents nonlinear interaction between the SCM channels.

The desired terms in this case are the ones with frequency $f_{r, k}$. Their amplitude can be maximized by setting $\Delta \phi_{r, k}=$ $(-1 / 2) \pi$. The terms with even multiples of the carrier frequencies will then disappear. Hence, each bandpass filter can select a particular SCM channel and suppress all other channels, provided that odd multiples of the carrier frequencies of the other channels are well outside the passband of the filter. In case of minimum channel spacing, this implies that the carrier frequencies of the SCM channels should be chosen such that the maximum carrier frequency is less than three times larger than the minimum carrier frequency.

When the bandpass filters are dimensioned such that the $j$ th filter passes the RF signal with carrier frequency $f_{r, j}$ and suppresses signals with other frequencies, then its expected output signal can be written as

$$
\begin{aligned}
E\left[V_{\mathrm{BP}, r, j}(t)\right]= & \frac{Z_{\mathrm{TIA}} R_{\mathrm{pd}} P_{\mathrm{in}}}{2 L_{\mathrm{Tx}} L_{\mathrm{Rx}} L_{\mathrm{nw}}}\left|H_{\mathrm{BP}, r, j}\left(f_{r, j}\right)\right| \\
& \cdot J_{1}\left(\beta_{r, j}(t)\right) \sin \left(2 \pi f_{r, j} t+\psi_{r, j}(t)\right) .
\end{aligned}
$$

Note that this expression is similar to (5). Therefore, $\beta_{i, k}(t)$ should be optimized in the same way as $\beta_{i}(t)$ in the case without SCM, resulting in a similar output signal. The main difference is that the network losses $L_{\mathrm{nw}}$ will now be $K$ times larger because an $N \cdot K$-port combiner is needed instead of an $N$-port combiner, so that the output signal will be $K$ times smaller. When the SCM channels in one CM channel are demultiplexed by $K$ separate CM receivers with one bandpass filter each (instead of one CM receiver with $K$ bandpass filters), then the output signal will be another $K$ times smaller.

Phase synchronization $\left(\Delta \phi_{r, k}=-(1 / 2) \pi\right)$ is not straightforward: If this was to be done in the same way as described in Section III-C, this would require all transmitter PDDs $T_{\mathrm{Tx}, i, k}$ in the same $\mathrm{CM}$ channel $i$ to be exactly the same. In case of significant fabrication inaccuracies, temperature sensitivity, or component aging, this can only be realized in case some kind of "master" transmitter provides synchronization signals in the return path, as described in [21]. This will not be considered in further detail here. In case the SCM channels are separately detected by different $\mathrm{CM}$ receivers, the transmitters corresponding to the same $\mathrm{CM}$ channel no longer need to have exactly the same PDD, but each receiver still needs to have a fixed phase relation to the corresponding transmitter: $\Delta \phi_{r, k}=2 \pi f_{\mathrm{c}}\left(T_{\mathrm{Rx}, r, k}-T_{\mathrm{Tx}, r, k}\right)=-(1 / 2) \pi, \forall k$, where $T_{\mathrm{Rx}, r, k}$ is the PDD of the CM receiver corresponding to the $k$ th SCM channel in the $r$ th CM channel. However, this phase synchronization cannot be obtained in the same way as described in Section III-C: The output signal of the low-pass filter would consist of several terms, corresponding to all SCM channels in that CM channel, and it is not possible to distinguish between those terms, so it does not provide a suitable control signal. A solution is described in [18].

The effect of chromatic dispersion and noise can be calculated in the same way as in the previous section. It will result in a CNR that is given by (15) [18], where

$$
\begin{aligned}
\mathrm{CNR}_{\mathrm{bn}} & =\frac{2\left\langle J_{1}^{2}\left(\beta_{r}(t)\right)\right\rangle\left|H_{\mathrm{CD}}\left(f_{r}\right)\right|^{2}}{\left(4 N^{2} K^{2}+(2 N-1) K\right) \tau_{\mathrm{c}} W_{\mathrm{BP}}} \\
\mathrm{CNR}_{\mathrm{sn}} & =\frac{R_{\mathrm{pd}} P_{\mathrm{in}}\left\langle J_{1}^{2}\left(\beta_{r}(t)\right)\right\rangle\left|H_{\mathrm{CD}}\left(f_{r}\right)\right|^{2}}{8 N K L_{\mathrm{Tx}} L_{\mathrm{Rx}} L_{\mathrm{nw}} e W_{\mathrm{BP}}} .
\end{aligned}
$$

$\mathrm{CNR}_{\mathrm{tn}}$ is still given by (18), and $\left|H_{\mathrm{CD}}\left(f_{r}\right)\right|^{2}$ is still given by (8). Note that substituting $K=1$ gives the same results as in the previous section.

As an example, suppose that single-channel SCM is used on top of CM in the downlink of the wireless LAN distribution network that was considered in Section III-F in order to distinguish between RAPs that share the same CM channel. Since three nonoverlapping channels are defined for IEEE 802.11b [1], we assume that three SCM channels are multiplexed per CM channel so that $K=3$. The distribution network now contains $3 N$ coherence modulators and $3 N$ coherence demodulators, so that the total network loss now becomes $L_{\mathrm{nw}} \approx 1.09 \cdot 9 N^{2} \approx$ $9.81 N^{2}$. The other network parameters are assumed to be the same as in the previous example. The resulting CNR is again calculated as a function of the number of CM channels and is shown in Fig. 5. 


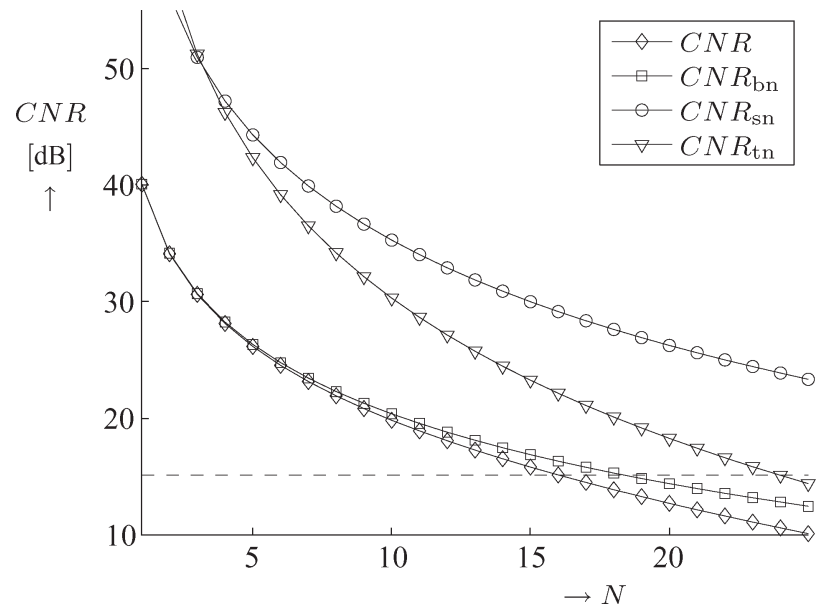

Fig. 5. Carrier-to-noise ratios at the output of the bandpass filter as a function of the number of CM channels $N$ in a wireless LAN IEEE $802.11 \mathrm{~b}$ distribution network based on CM and single-channel SCM, with three SCM channels per CM channel.

By maintaining the $15-\mathrm{dB}$ criterion, it follows that, at most, there are $16 \mathrm{CM}$ channels, and hence, $3 \cdot 16=48$ RAPs can be supported, which is exactly the same as in the previous example, without SCM. This can be explained by the fact that coherence modulators with identical PDDs cause approximately the same amount of beat noise as an identical number of coherence modulators with different PDDs. The same goes for the shot and thermal noises because the losses are still the same as in the previous case.

If the same concept were used in the uplink, where the receivers for the different SCM channels in one $\mathrm{CM}$ channel can be combined into one $\mathrm{CM}$ receiver, this would result in reduced optical losses and, hence, improved carrier-to-shot and carrierto-thermal noise ratios. The same would be the case if SCM was used to distinguish between three MTs that are simultaneously supported through the same RAP in the downlink.

\section{B. Multichannel SCM}

Now, suppose that $K \mathrm{RF}$ signals are transmitted through the same CM channel $i$ by means of multichannel SCM. The SCM channels, which are denoted by (19), are then first combined in the electrical domain, resulting in a composite signal

$$
\phi_{\text {mod }, i}(t)=\sum_{k=1}^{K} \beta_{i, k}(t) \sin \left(2 \pi f_{i, k} t+\psi_{i, k}(t)\right) .
$$

When $N$ of such composite signals are coherence-multiplexed using a similar system, as described in Section III-A, the output signal of the $j$ th bandpass filter of $\mathrm{CM}$ receiver $r$ can be calculated by means of (2). By using

$$
\begin{aligned}
& \exp \left(j \sum_{k=1}^{K} \beta_{k} \sin \Phi_{k}\right) \\
& =\sum_{n_{1}} \sum_{n_{2}} \cdots \sum_{n_{K}}\left[\prod_{k=1}^{K} J_{n_{k}}\left(\beta_{k}\right)\right] \exp \left(j \sum_{k=1}^{K} n_{k} \Phi_{k}\right)
\end{aligned}
$$

this can be shown to result in

$$
\begin{aligned}
& E\left[V_{\mathrm{BP}, r, j}(t)\right]=\frac{Z_{\mathrm{TIA}} R_{\mathrm{pd}} P_{\mathrm{in}}}{4 L_{\mathrm{Tx}} L_{\mathrm{Rx}} L_{\mathrm{nw}}} \\
& \cdot \int h_{\mathrm{BP}, r, j}(t-\rho) \sum_{n_{1}} \sum_{n_{2}} \cdots \sum_{n_{K}}\left[\prod_{k=1}^{K} J_{n_{k}}\left(\beta_{r, k}(\rho)\right)\right] \\
& \cdot\left\{\cos \left(\Delta \phi_{r}\right) \cos \left(\sum_{k=1}^{K} n_{k}\left[2 \pi f_{r, k} \rho+\psi_{r, k}(\rho)\right]\right)-\sin \left(\Delta \phi_{r}\right)\right. \\
& \left.\cdot \sin \left(\sum_{k=1}^{K} n_{k}\left[2 \pi f_{r, k} \rho+\psi_{r, k}(\rho)\right]\right)\right\} d \rho .
\end{aligned}
$$

Apparently, IMD occurs between the SCM channels due to the intrinsic nonlinearity of the demodulation principle. As a result, the output signal contains one baseband term (that is the term for which $n_{1}=n_{2}=\cdots=n_{K}=0$ ) and an infinite sum of RF terms with carrier frequencies that are linear combinations of the carrier frequencies $f_{r, k}$ of the SCM channels. The desired RF signal term is the one with carrier frequency $f_{r, j}$ and phase $\psi_{r, j}(t)$, i.e., the one with $n_{j}= \pm 1$ and $n_{K}=0$ for $k \neq j$. Its amplitude can again be maximized by setting $\Delta \phi_{r}=-(1 / 2) \pi$ (which can be done in the same way as described in Section III-C). The bandpass filter should be dimensioned such that this term is passed and the other terms are suppressed, resulting in a desired output signal part

$$
\begin{aligned}
E\left[V_{\mathrm{BP}, r, j}(t)\right]_{\mathrm{desired}}= & \frac{Z_{\mathrm{TIA}} R_{\mathrm{pd}} P_{\mathrm{in}}}{2 L_{\mathrm{Tx}} L_{\mathrm{Rx}} L_{\mathrm{nw}}}\left|H_{\mathrm{BP}, r, j}\left(f_{r, j}\right)\right| \\
& \cdot\left[\prod_{\substack{k=1 \\
k \neq j}}^{K} J_{0}\left(\beta_{r, k}(t)\right)\right] \cdot J_{1}\left(\beta_{r, j}(t)\right) \\
& \cdot \sin \left(2 \pi f_{r, j} t+\psi_{r, j}(t)\right) .
\end{aligned}
$$

Note that this requires the amplitudes $\beta_{r, k}(t)$ of the SCM channels to be small $(\ll 1)$ so that variations due to interfering SCM channels (described by the zero-order Bessel functions) are negligible or-in case of constant amplitudes-to maximize the amplitude of the detected signal.

The remaining terms in (26) are all IMD products. For example, the terms that have $n_{k}= \pm 1$ for two different values of $k$, and all remaining $n_{k}$ s equal to zero are second-order IMD products. It can be verified that these terms cancel each other when $\Delta \phi_{r}=-(1 / 2) \pi$. The same goes for the terms having $n_{k}= \pm 2$ for only one value of $k$ and all remaining $n_{k}$ s equal to zero. Therefore, second-order IMD does not play a role in this system, irrespective of the frequency range that is used.

The terms that have $n_{k}= \pm 1$ for three different values of $k$ and all remaining $n_{k}$ s equal to zero are third-order IMD products. These do not cancel each other and appear at frequencies $\pm f_{r, k_{1}} \pm f_{r, k_{2}} \pm f_{r, k_{3}}$. These coincide with the desired RF frequencies when there are three or more SCM channels with equal differences between the carrier frequencies. In the case of constant SCM channel amplitudes $\beta$, the amplitudes of these 
terms are proportional to $J_{1}^{3}(\beta) J_{0}^{K-3}(\beta)$. We will, from now on, denote these as type I.

Similarly, the terms that have $n_{k_{1}}= \pm 2$ and $n_{k_{2}}= \pm 1$, with $k_{1} \neq k_{2}$, and all remaining $n_{k}$ s equal to zero are also thirdorder IMD products. These also do not cancel each other and appear at frequencies $2 f_{r, k_{1}} \pm f_{r, k_{2}}$. These might also coincide with the desired RF frequencies. In case of constant SCM channel amplitudes $\beta$, the amplitudes of these terms are proportional to $J_{2}(\beta) J_{1}(\beta) J_{0}^{K-2}(\beta)$. We denote these as type II.

Finally, the terms that have $n_{k}= \pm 3$ and all remaining $n_{k} \mathrm{~s}$ equal to zero are also third-order IMD products that do not cancel each other, appearing at frequencies $3 f_{r, k}$. These will not coincide with the desired RF frequencies if the RF frequencies are chosen in a sufficiently small bandwidth. In case of constant SCM channel amplitudes $\beta$, the amplitudes of these terms are proportional to $J_{3}(\beta) J_{0}^{K-1}(\beta)$. These are denoted as type III.

All other terms lead to higher order IMD effects. Since the amplitudes $\beta_{r, k}(t) \ll 1$ (as discussed earlier), the amplitudes of the higher order IMD products will be negligible. Therefore, IMD in a phase-synchronized CM receiver for multichannel $\mathrm{SCM}$ over CM is dominated by third-order IMD. The number of third-order IMD products that appear in the passband of the bandpass filter depends on the choice of the carrier frequencies $f_{r, k}$, and their amplitude depends on the modulation format of the SCM channels and the transfer function of the bandpass filter. When both the power spectral density of the modulated SCM channels and the transfer function of the bandpass filter are approximated as rectangular functions with identical bandwidth $W_{\mathrm{BP}}$ and center frequency $f_{r, j}$, then it can be shown that approximately two thirds of the power of a third-order IMD product is passed through by the bandpass filter [22] Hence, when it is assumed that the SCM channel amplitudes are a constant $\beta$ and that only a negligible power of the thirdorder IMD products leaks into adjacent channels, then the carrier-to-intermodulation ratio (CIR) follows from (27) and the aforementioned amplitudes as

$$
\begin{aligned}
\operatorname{CIR}_{\mathrm{IMD}} \triangleq & \frac{\left\langle\mathrm{E}^{2}\left[V_{\mathrm{BP}, r, j}(t)\right]_{\text {desired }}\right\rangle}{\left\langle\mathrm{E}^{2}\left[V_{\mathrm{BP}, r, j}(t)\right]_{\mathrm{IMD}}\right\rangle} \\
=3 J_{0}^{4}(\beta) J_{1}^{2}(\beta) & {\left[K_{\mathrm{I}} J_{1}^{6}(\beta)+K_{\mathrm{II}} J_{0}^{2}(\beta) J_{1}^{2}(\beta) J_{2}^{2}(\beta)\right.} \\
& \left.\quad+K_{\mathrm{III}} J_{0}^{4}(\beta) J_{3}^{2}(\beta)\right]^{-1}
\end{aligned}
$$

where $K_{\mathrm{I}}, K_{\mathrm{II}}$, and $K_{\mathrm{III}}$ are the number of terms of the form types I, II, and III, respectively, that appear at center frequency $f_{r, j}$.

The effect of chromatic dispersion and noise can be calculated in the same way as in the previous section. In case of constant SCM channel amplitudes, this can be shown to result in a carrier-to-noise and intermodulation ratio

$$
\begin{aligned}
\mathrm{CNIR} & \triangleq \frac{\left\langle\mathrm{E}^{2}\left[V_{\mathrm{BP}, r, j}(t)\right]_{\mathrm{desired}}\right\rangle}{\sigma_{V_{\mathrm{BP}, r}(t)}^{2}+\left\langle\mathrm{E}^{2}\left[V_{\mathrm{BP}, r, j}(t)\right]_{\mathrm{IMD}}\right\rangle} \\
& =\left(\mathrm{CNR}_{\mathrm{sn}}^{-1}+\mathrm{CNR}_{\mathrm{bn}}^{-1}+\mathrm{CNR}_{\mathrm{tn}}^{-1}+\mathrm{CIR}_{\mathrm{IMD}}^{-1}\right)^{-1}
\end{aligned}
$$

where

$$
\begin{aligned}
\mathrm{CNR}_{\mathrm{bn}} & =\frac{2 J_{0}^{2 K-2}(\beta) J_{1}^{2}(\beta)\left|H_{\mathrm{CD}}\left(f_{r}\right)\right|^{2}}{\left(4 N^{2}+2 N-1\right) \tau_{\mathrm{c}} W_{\mathrm{BP}}} \\
\mathrm{CNR}_{\mathrm{sn}} & =\frac{R_{\mathrm{pd}} P_{\mathrm{in}} J_{0}^{2 K-2}(\beta) J_{1}^{2}(\beta)\left|H_{\mathrm{CD}}\left(f_{r}\right)\right|^{2}}{8 N L_{\mathrm{Tx}} L_{\mathrm{Rx}} L_{\mathrm{nw}} e W_{\mathrm{BP}}} \\
\mathrm{CNR}_{\mathrm{tn}} & =\frac{R_{\mathrm{pd}}^{2} P_{\mathrm{in}}^{2} J_{0}^{2 K-2}(\beta) J_{1}^{2}(\beta)\left|H_{\mathrm{CD}}\left(f_{r}\right)\right|^{2}}{16 L_{\mathrm{Tx}}^{2} L_{\mathrm{Rx}}^{2} L_{\mathrm{nw}}^{2} S_{\mathrm{th}} W_{\mathrm{BP}}} .
\end{aligned}
$$

$\mathrm{CIR}_{\mathrm{IMD}}$ is given by (28), and $\left|H_{\mathrm{CD}}\left(f_{r}\right)\right|^{2}$ is still given by (8). Note that substituting $K=1$ gives the same results as in Section III.

Choosing $\beta$ is now a tradeoff: Choosing it too low gives a low CNR as it results in a small desired signal, whereas choosing it too large gives a low CIR as it results in a large IMD, so there is an optimal value for $\beta$, which depends on the other network parameters.

Again, consider the numerical example of the previous section, but now with multichannel instead of single-channel SCM. The European center frequencies for the three nonoverlapping channels in IEEE $802.11 \mathrm{~b}$ are given by $f_{1}=2412 \mathrm{MHz}, f_{2}=$ $2442 \mathrm{MHz}$, and $f_{3}=2472 \mathrm{MHz}$. Since these frequencies satisfy $2 f_{2}-f_{3}=f_{1}, f_{1}-f_{2}+f_{3}=f_{2}$, and $2 f_{2}-f_{1}=f_{3}$, it follows that $K_{\mathrm{I}}=K_{\mathrm{III}}=0$ and $K_{\mathrm{II}}=2$ for channels 1 and 3 , and $K_{\mathrm{I}}=2$ and $K_{\mathrm{II}}=K_{\mathrm{III}}=0$ for channel 2. By using (28), it can be verified that channel 2 has the lowest CIR, which is given by

$$
\mathrm{CIR}_{\mathrm{IMD}}=\frac{3}{2}\left(\frac{J_{0}(\beta)}{J_{1}(\beta)}\right)^{4} .
$$

Therefore, we will, from now on, only consider channel 2 . The CM network now contains $N$ coherence modulators and $3 N$ coherence demodulators, so that the total network loss now becomes $L_{\mathrm{nw}} \approx 1.09 \cdot 3 N^{2} \approx 3.27 N^{2}$. The other network parameters are assumed to be the same as before. The resulting CNIR is calculated as a function of $\beta$ and the number of CM channels $N$, optimized with respect to $\beta$ for each value of $N$, and is shown in Fig. 6(a). The corresponding optimum value of $\beta$ is shown in Fig. 6(b). Note that the optimum value of $\beta$ increases with an increasing $N$. This is because an increasing $N$ increases the loss and the number of beat noise terms, resulting in a decreased CNR if the value of $\beta$ were fixed. Therefore, $\beta$ has to be increased in order to maintain a certain CNR, although it comes with a decrease in CIR.

By maintaining the 15-dB criterion, it follows that, at most, there are $19 \mathrm{CM}$ channels, and hence, $3 \cdot 19=57$ RAPs can be supported, which is slightly more than in the previous examples, without SCM or with single-channel SCM. This can be explained by the fact that fewer coherence modulators are required for transmission in all the channels, so that there are less beat noise terms and lower optical losses. Apparently, this fully compensates for the performance degradation due to IMD.

\section{CONCLUSION}

It has been proposed that $\mathrm{CM}$ can be used as an addressing scheme in an optical RF feeding network where RF signals 


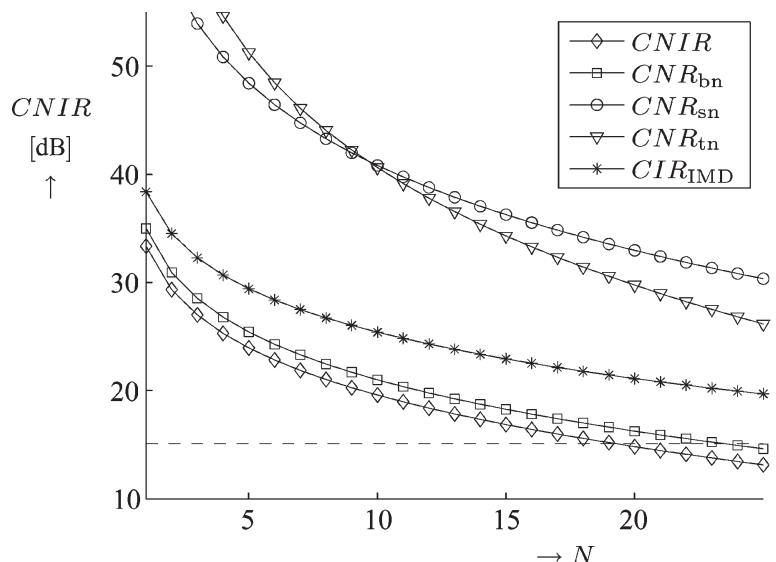

(a)

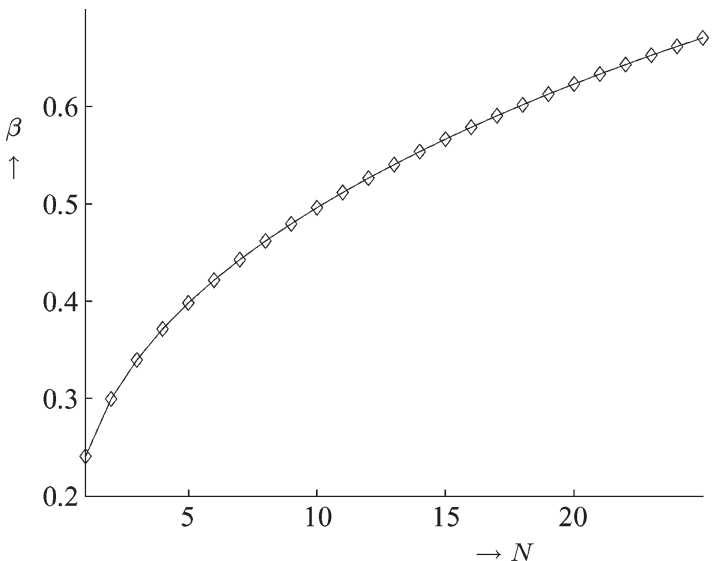

(b)

Fig. 6. Carrier-to-noise and intermodulation ratios at the output of the bandpass filter as a function of the number of CM channels $N$ in a wireless LAN IEEE $802.11 \mathrm{~b}$ distribution network based on CM and multichannel SCM, with three SCM channels per CM channel, and optimized modulation index $\beta$. (a) Maximum CNIRs. (b) Optimized modulation index $\beta$.

are distributed to multiple RAPs. The inherent nonlinearity of the demodulation process in a CM system will result in harmonic distortion, so that the modulation depth should be kept small (if the amplitude of the RF signal is modulated) or optimized (in case of constant or ON/OFF-modulated RF signal amplitude). The output signal can be stabilized by a relatively simple feedback loop, which does not require frequency dithering.

The impact of chromatic dispersion has been described and analyzed. In a CM distribution system for IEEE $802.11 \mathrm{~b}$ wireless LAN, with a network span of $500 \mathrm{~m}$ (using standard SMF), and optical sources with a $1550-\mathrm{nm}$ center wavelength and 0.1-ps coherence time, a dramatic dispersion penalty of approximately $50 \mathrm{~dB}$ would result, whereas center wavelengths of $1310 \mathrm{~nm}$ would result in a negligible dispersion penalty (below $0.2 \mathrm{~dB}$ ).

From noise performance analysis, it follows that approximately 48 RAPs can simultaneously be supported in such a network, satisfying a minimum CNR of $15 \mathrm{~dB}$.

Multiple RF channels can be multiplexed into one CM channel by using SCM on top of CM. It has been explained that this can be performed either by transmitting multiple optical carriers in the same CM channel, where each contains one SCM channel (single-channel SCM), or by first combining the SCM channels in the electrical domain and modulating the composite signal onto one CM channel (multichannel SCM).

Single-channel SCM has the advantage that it can be used when the different SCM channels are generated in different transmission nodes. Each coherence modulator still introduces a comparable amount of beat and shot noises so that noise performance is comparable to the case where each RF signal is transmitted in a separate CM channel.

The advantage of multichannel SCM is that less coherence modulators are required, which reduces the costs and network losses. However, it can only be used when all the SCM channels in one CM channel are generated in the same node. The main disadvantage is that IMD occurs due to the nonlinearity of coherence modulation and demodulation. Although the amount of beat noise is reduced due to the reduced number of coherence modulators, IMD requires the modulation index to be reduced to such a value such that the resulting noise performance is comparable to the single-channel SCM case.

An interesting property of $\mathrm{CM}$ is that it is transparent, in the sense that the channels can independently operate. Hence, CM can be applied in a system where both digital and analog signals are distributed, supporting fixed terminals at high speeds and MTs at relatively low speeds.

IEEE $802.11 \mathrm{~b}$ was considered as an example of a wireless transmission standard that can be supported by optical RF distribution. Alternatively, a higher data rate system such as IEEE 802.11g [2] could also be considered, but this has the problem that 52 OFDM carriers are used per MT, which requires a large number of SCM channels per CM channel. This will introduce either large network losses and beat noise power (singlechannel SCM) or a considerable IMD (multichannel SCM). The latter could be reduced by using predistortion techniques prior to optical modulation.

Chromatic dispersion can become an issue at higher radio frequencies: By using the theory from Section III-D and assuming the same parameter values as in Section III-F, one can derive dispersion penalties of $0.7 \mathrm{~dB}$ at $5 \mathrm{GHz}, 2.5 \mathrm{~dB}$ at $11 \mathrm{GHz}$, and $10.2 \mathrm{~dB}$ at $66 \mathrm{GHz}$. This could be ameliorated by means of low-dispersion fiber or dispersion compensation techniques.

\section{ACKNOWLEDGMENT}

The authors would like to thank Dr. C. G. H. Roeloffzen for the helpful discussions on integrated optics technology and Prof. J. C. Haartsen for providing information on wireless LAN technology.

\section{REFERENCES}

[1] Supplement to IEEE Standards for Information TechnologyTelecommunications and Information Exchange Between Systems-Local and Metropolitan Area Networks-Specific Requirements-Part 11: Wireless LAN Medium Access Control (MAC) and Physical Layer (PHY) Specifications: Higher Speed Physical Layer Extension in the $2.4 \mathrm{GHz}$ Band, IEEE Std. 802.11b, 1999 
[2] Supplement to IEEE Standards for Information TechnologyTelecommunications and Information Exchange Between Systems-Local and Metropolitan Area Networks-Specific Requirements-Part 11: Wireless LAN Medium Access Control (MAC) and Physical Layer (PHY) Specifications: Further Higher Data Rate Extension in the $2.4 \mathrm{GHz}$ Band, IEEE Std. 802.11g, 2003

[3] H. Al-Raweshidy and S. Komaki, Radio Over Fiber Technologies for Mobile Communications Networks. Norwood, MA: Artech House, 2002.

[4] C. Delisle and P. Cielo, "Application de la modulation spectrale à la transmission de l'information," Can. J. Phys., vol. 53, pp. 1047-1053, 1975.

[5] P. Cielo and C. Delisle, "Multiplexage en communication optique par interférometrie à grande différence de marche en lumière blanche," Can. J. Phys., vol. 54, pp. 2322-2331, 1976.

[6] J. L. Brooks, R. H. Wentworth, R. C. Youngquist, M. Tur, B. Y. Kim, and H. J. Shaw, "Coherence multiplexing of fiber-optic interferometric sensors," J. Lightw. Technol., vol. LT-3, no. 5, pp. 1062-1072, Oct. 1985.

[7] J.-P. Goedgebuer, H. Porte, and A. Hamel, "Electrooptic modulation of multilongitudinal mode laser diodes: Demonstration at $850 \mathrm{~nm}$ with simultaneous data transmission by coherence multiplexing," IEEE $J$. Quantum Electron., vol. QE-23, no. 7, pp. 1135-1144, Jul. 1987.

[8] J.-P. Goedgebuer and A. Hamel, "Coherence multiplexing using a parallel array of electrooptic modulators and multimode semiconductor lasers," IEEE J. Quantum Electron., vol. QE-23, no. 12, pp. 2224-2237, Dec. 1987.

[9] R. H. Wentworth, "Theoretical noise performance of coherencemultiplexed interferometric sensors," J. Lightw. Technol., vol. 7, no. 6, pp. 941-956, Jun. 1989.

[10] K. W. Chu and F. M. Dickey, "Optical coherence multiplexing for interprocessor communications," Opt. Eng., vol. 30, no. 3, pp. 337-344, Mar. 1991.

[11] H. Uehara and I. Sasase, "Coherence multiplexed subcarrier multiplexing (CM/SCM) lightwave system for microcelluler mobile communications," IEICE Trans. Commun., vol. E79-B, no. 5, pp. 708-715, May 1996.

[12] H. Uehara, I. Sasase, and M. Yokoyama, "Fiber optic subcarrier transmission systems using coherence multiplexing techniques for broad-band distribution networks," IEICE Trans. Commun., vol. E80-B, no. 7, pp. 1027-1034, Jul. 1997.

[13] G. J. Pendock and D. D. Sampson, "Capacity of coherence-multiplexed CDMA networks," Opt. Commun., vol. 143, no. 1-3, pp. 109-117, Nov. 1997.

[14] J. Kani, K. Iwatsuki, N. Takachio, and N. Fujii, "A simple broad-band coherence multiplexed optical access network and its scalability," J. Lightw. Technol., vol. 19, no. 4, pp. 456-464, Apr. 2001.

[15] R. O. Taniman, A. Meijerink, W. van Etten, and J. C. Haartsen, "Indoor RF signal distribution using a coherence multiplexed/subcarrier multiplexed optical transmission system," in Proc. 10th IEEE/CVT Symp. Benelux, Eindhoven, The Netherlands, Nov. 2003, p. 17.

[16] A. Meijerink, G. H. L. M. Heideman, and W. van Etten, "Balanced optical phase diversity receivers for coherence multiplexing," J. Lightw. Technol., vol. 22, no. 11, pp. 2393-2408, Nov. 2004.

[17] A. Meijerink, "Coherence multiplexing for optical communication systems," Ph.D. dissertation, Univ. Twente, Enschede, The Netherlands, Oct. 2005. [Online]. Available: http://doc.utwente.nl/50841/

[18] A. Meijerink, R. O. Taniman, G. H. L. M. Heideman, and W. van Etten, "Coherence multiplex system topologies," IEEE J. Sel. Topics Quantum Electron., vol. 13, no. 5, Sep./Oct. 2007, to be published.

[19] J. L. Gimlett and N. K. Cheung, "Dispersion penalty analysis for LED/single-mode fiber transmission systems," J. Lightw. Technol., vol. LT-4, no. 9, pp. 1381-1392, Sep. 1986.

[20] Int. Telecommun. Union, Characteristics of a Single-Mode Optical Fibre and Cable, 2003. ITU-T Recommendation G.652.

[21] R. A. Griffin, D. D. Sampson, and D. A. Jackson, "Optical-phase coding for code-division multiple access networks," IEEE Photon. Technol. Lett., vol. 4, no. 12, pp. 1401-1404, Dec. 1992.

[22] Y.-H. Lee, J. Wu, and H.-W. Tsao, "The impact of laser phase noise on the coherent subcarrier multiplexing system," J. Lightw. Technol., vol. 9, no. 3, pp. 347-355, Mar. 1991.

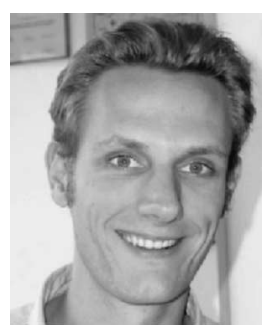

Arjan Meijerink (S'00-M'06) was born in Almelo, The Netherlands, in 1976. He received the M.Sc. and $\mathrm{Ph} . \mathrm{D}$. degrees (both with honors) in electrical engineering from the University of Twente, Enschede, The Netherlands, in 2001 and 2005, respectively.

In 2000, he was a Guest Researcher at Ericsson Business Mobile Networks, Enschede, developing error concealment techniques for Bluetooth voice links. From 2001 to 2005, he carried out his Ph.D. research on optical coherence multiplexing with the Telecommunication Engineering Group, University of Twente, where he is currently a Postdoctoral Researcher. His current research interests include optical communications and RF photonic signal processing techniques, particularly optical beam forming.

Dr. Meijerink received the Gauss Award for the best young researcher's presentation during the 22nd Symposium on Information and Communication Theory in the Benelux in May. He was awarded the second prize in the IEEE Region 8 Student Paper Contest on July 2001.

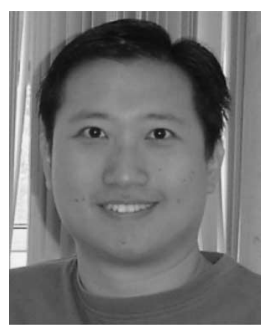

Robert O. Taniman (S'06) was born in Surabaya, Indonesia, in 1974. He received the B.Sc. degree (with honors) in engineering physics from the Institut Teknologi Sepuluh Nopember, Surabaya, in 1998 and the M.Sc. degree (with honors) in electrical engineering from the University of Twente, Enschede, The Netherlands, in 2003, where he is currently working toward the Ph.D. degree with the Chair for Design and Analysis of Communication Systems.

Between 1998 and 2001, he was an Assistant Lecturer with the University of Surabaya. His research includes the design and development of a resource allocation strategy for a multicarrier multimode passive optical network deployed as a broadband access network.

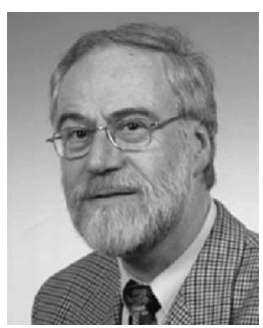

Wim van Etten (M'80-SM'91) was born in Zevenbergen, The Netherlands, in 1942. He received the M.Sc. and Ph.D. degrees in electrical engineering from Eindhoven University of Technology, Eindhoven, The Netherlands, in 1969 and 1976, respectively.

From 1969 to 1970, he was with Philips Electronics, developing circuits for oscilloscopes. In 1970, he became an Assistant Professor with the Faculty of Electrical Engineering, Eindhoven University of Technology. From 1970 to 1976 , he was engaged in research on the transmission of digital signals via coaxial and multiwire cables. Since 1976, he has been involved with research and education on optical fiber communications. In 1985, he was appointed as an Associate Professor with Eindhoven University of Technology. In 1994, he became a Full Professor of telecommunications with the University of Twente, Enschede, The Netherlands. His current interests include optical communications, mobile communications, detection, and simulation of communication systems. He is the author or coauthor of more than 80 papers in international journals, conferences, and symposia. He is the first author of Fundamentals of Optical Fiber Communications (Prentice-Hall, 1991) and the author of Introduction to Random Signals and Noise (Wiley, 2005). Two of his papers were selected for a collection of 57 key papers on communications that appeared in the last 50 years in IEEE ComSoc publications.

Prof. van Etten is a member of the Dutch Institute of Electronic and Radio Engineers. He is the Chairman of the executive committee of the IEEE Benelux joint Chapter on Communications and Vehicular Technology. He has been a member of organizing committees and program committees of various conferences and symposia. 